\title{
Le vivre et mourir en ligne du Vivre ensemble de Roland Barthes
}

\section{Guillaume Bellon}

\section{Q OpenEdition}

12 Journals

Édition électronique

URL : http://journals.openedition.org/recherchestravaux/109

DOI : 10.4000/recherchestravaux.109

ISSN : 1969-6434

Éditeur

UGA Éditions/Université Grenoble Alpes

\section{Édition imprimée}

Date de publication : 15 juin 2008

Pagination : $217-226$

ISBN : 978-2-84310-125-0

ISSN : 0151-1874

\section{Référence électronique}

Guillaume Bellon, "Le vivre et mourir en ligne du Vivre ensemble de Roland Barthes », Recherches \& Travaux [En ligne], 72 | 2008, mis en ligne le 15 décembre 2009, consulté le 03 février 2021. URL: http://journals.openedition.org/recherchestravaux/109; DOI : https://doi.org/10.4000/ recherchestravaux.109 
Guillaume BELLON

Université Stendhal - Grenoble 3

\section{Le vivre et mourir en ligne du Vivre ensemble de Roland Barthes}

Soit la liste suivante : «Computer, electric, computer game, computer memory, magnetic tape, computer rental, computer network, computer support, samsung, electrical appliance, notebook computer.» Derrière cet inventaire, il ne s'agit pas de retrouver quelque chose comme «une certaine encyclopédie chinoise»-celle-là même que cite Foucault à l'ouverture des Mots et les choses ${ }^{\mathrm{I}}$ - qui nous confronterait à quelque limite de notre entendement, mais de constater l'absence d'un site. Le site www.roland-barthes.com, ouvert à l'automne 2003 , n'existe plus ${ }^{2}$, et en lieu et place, on trouve l'étoilement des items mentionnés plus haut.

\section{L'ultime mythologie de l'œuvre?}

Que nous reste-t-il, dès lors, face à ce qui n'est plus, quand du passé rien ne subsiste, après la destruction des choses, si ce n'est le discours de l'hommage? Cet hommage, je voudrais m'en acquitter par le détour d'une parodie, ou plus exactement, d'un pastiche des Mythologies : m'inspirant non de Barthes luimême, mais d'Umberto Eco, pour qui «la parodie se veut aussi hommage ${ }^{3} »$, j'imaginerai alors que l'œuvre de Barthes, dans son devenir et ses multiples mises en publicité, nous offre une ultime mythologie, qui serait justement celle

I. M. Foucault, Les Mots et les choses, Une archéologie des sciences humaines, Gallimard, I966, p. 7- I6.

2. Voir sur ce point l'article de Cl. Coste dans le présent volume, p. $20 \mathrm{I}$.

3. U. Eco, Pastiches et postiches, trad. de l'italien par B. Guyader, Messidor, I 988. 
de la mise en ligne du premier cours au Collège de France, dispensé par Barthes en 1977, Comment vivre ensemblet. Cette mise en ligne, dans le droit fil d'une publication problématique (il s'agit de «Notes de cours» présentant des états de rédaction variés et des abréviations), inviterait ainsi à interroger l'interaction de l'œuvre avec ses lecteurs. En effet, dans cette mythologie que je pastiche grossièrement, je voudrais déceler les traces visibles, les arcanes d'un récit primaires. Tout serait réuni, à commencer par les difficultés initiales, véritables péripéties à rebondissement : il y eut la réticence du premier cercle des fidèles, se prévalant de leur fréquentation du maitre pour se faire garants du respect de sa pensée et de son intégrité intellectuelle; puis il y eut ce qu'on désigne discrètement aujourd'hui comme «l'affaire Règle du jeu». En août I 99I, la revue La Règle du jeu, dirigée par Bernard-Henri Lévy, propose une transcription non autorisée de la quasi-intégralité de la première séance du Neutre, le cours tenu par Barthes en 1978 (soit une année après notre Vivre ensemble). Plus préoccupée sans doute de créer l'événement qu'animée par un souci scientifique ou éthique de fidélité au propos de Barthes au Collège de France, cette publication choque par sa médiocrité ${ }^{6}$. Attaquée en justice, la revue est condamnée, le 20 novembre I99I, à payer cinquante mille francs de dommages et intérêts à Michel Salzedo, héritier de Roland Barthes, pour «avoir porté atteinte au droit de divulgation qu'il possède sur l'œuvre» de son demifrère ${ }^{7}$. Passé le rebondissement judiciaire, une fois les querelles apaisées, prit alors place la longue attente, qui verrait notre petit récit emprunter au sommeil réparateur des contes de fées, pour assurer enfin, à l'automne 2002, l'aboutissement de la quête (celle de ces cours devenus saint graal), par une double

\footnotetext{
4. Le cours est édité par Cl. Coste, sous la direction d'É. Marty, sous le titre : Comment vivre ensemble: simulations romanesques de quelques espaces quotidiens, Seuil/IMEC, «Traces écrites», 2002. 5. Si peu de mythologies retracent le fil narratif d'un événement ou d'une masse discursive envisagée dans sa logique narrative, on notera que Barthes relève le «spectacle» du «Monde où l'on catche» (p. 679-688), les «fantaisies créatives» de «La croisière de sang bleu» (p. 695697), la «légende implicite» de l'eau de Javel dans «Saponides et détergents» (p. 699-700). Bien évidemment, la tentative ici menée trouve son modèle dans «Le Tour de France comme épopée» (p. 756-765), et c'est, en outre, à la «Cuisine ornementale» (p. 770-772) que j'emprunte l'emploi de la «fable» qui est fait ici. Voir Mythologies, reprises dans les CEurres complètes éditées par É. Marty, Seuil, 2002 (désormais abrégées $O C$ ), t. I.

6. Voir L. Dispot, «Roland Barthes : Le Désir de neutre», La Règle du Jeu, nº 5, août I 99I, p. 36-6o.

7. Voir S. de Faultrier-Travers, Le Droit d'auteur dans l'édition, Imprimerie nationale, I 993 : «[...] les récentes affaires Barthes, Lacan, Foucault, mettent également en scène ce droit de faire paraittre des cours alors même que leur auteur, mort, ne peut valider le travail de transcription, ni exercer son contrôle de destination. Se joue ici à nouveau l'apparent conflit entre le droit de l'œuvre d'accéder à une large diffusion, sous-tendu par le droit du public d'accéder à une large diffusion, et le droit privatif de l'auteur puis de ses ayants-droit d'apprécier l'opportunité d'une publication» (p. 57).
} 
actualité éditoriale : l'édition papier du Vivre ensemble et du Neutre ${ }^{8}$, assortie des enregistrements sonores correspondants, sous forme de CD MP 3 . L'aboutissement de la publication en ligne du premier cours, un an plus tard, marquerait une conjonction entre le sujet - le lecteur - et l'objet de son désir à la puissance quatre : le site proposait en effet le manuscrit original, en un splendide facsimilé, la transcription du texte de ce fac-similé, la transcription de la parole prononcée par Barthes, et l'enregistrement sonore. De quoi ravir, et c'était bien l'ambition du projet, le chercheur comme le lecteur ponctuel, qui verrait sa papillonne se doubler d'un certain voyeurisme, puisqu'il accéderait aux documents personnels de Barthes, aux brouillons de son travail. Mais l'histoire ne saurait en rester là, les temps heureux durer toujours : le coût prohibitif de l'abonnement, qui profilait l'accès au site pour les bibliothèques ou les centres de recherche seulement, fait que la logique financière se retourne contre elle-même, et, quatre années après son ouverture, le site est discrètement fermé, dans une indifférence quasi générale. Comme il se doit, le récit circulerait donc avec sa moralité, où l'on verrait, une fois de plus, l'art (ou plus modestement la littérature) aux prises avec le grand capital, lequel, aveugle et mesquin, n'a que faire des intérêts scientifiques, des découvertes théoriques... Si cette fable séduit tant, c'est qu'elle dit l'éphémérité de ce nouveau medium qu'est Internet, son possible illusionnisme, en un mot, sa fâcheuse tendance (à moins que ce ne soit sa tendance pour les fâcheux) à n'être que feu de paille : le vivre et mourir en ligne du Vivre ensemble ne serait que l'illustration de la validité temporaire de ces «textes numériques, éphémères, surabondants et hybrides ${ }^{9} »$.

Mais n'y a-t-il rien d'autre à lire, dans la clôture du roland-barthes.com, que la condamnation par provision de l'attrait suscité par les nouveaux media? Si l'expérience menée à l'occasion du site invite à interroger les effets de convergence entre théorique et technique (entre Texte et hypertexte), de même que leur possible dissension paraît l'occasion d'entrer dans le problème de la poétique effective du cours, il s'agira d'envisager la chance que représentait le recours au multimédia. L'offre simultanée du document établi pour l'édition papier (la transcription des notes de Barthes pour le Collège), du manuscrit autographe correspondant, de l'enregistrement sonore et de la transcription de celui-ci paraissait en effet la réponse adaptée à cette entreprise singulière autant que délicate qui consiste à faire de l'énonciation du cours un énoncé.

8. Le Neutre, édité par'Th. Clerc, sous la direction d’É. Marty, Seuil/IMEC, «Traces écrites», 2002.

9. J.-M. Salaün et Chr. Vandendorpe, «Introduction» aux Défis de la publication sur le web: byperlectures, cybertextes et méta-éditions, Villeurbanne, Presses de l'ENSSIB, 2004, p. I 3. 


\section{De la convergence entre théorique et technique}

C'est peu dire, tout d'abord, que l'œuvre de Barthes semble s'offrir d'ellemême à la technologie hypertextuelle. Tout se joue, en effet, autour de l'hypertexte, qu'on a tôt fait d'envisager comme version ultime d'un intertexte dont on sait ce qu'il doit à Barthes. On connaît les nombreuses définitions du Texte (alors sanctionné par la majuscule) qu’a proposées le critique dans les années I 970, dans le sillage d'une Kristeva fidèle lectrice de Bakhtine. Non pas «produit», mais «productivitér ${ }^{10} »$, non pas «structure», mais «structuration», le Texte «est un travail et un jeu»; pour le dire en un mot qui expliquera toute l'ambiguité : «c'est un volume de traces en déplacement ${ }^{1 \mathrm{I}} »$. On comprend que cette définition d'un texte comme activité incessante de production de sens, dans l'écriture comme dans la lecture (laquelle d'ailleurs est écriture à son tour), rencontre heureusement la technologie de l'hypertexte. Il n'est que de se souvenir de l'ouvrage de George Landow, Hypertext: the convergence of contemporary critical theory and technology ${ }^{12}$, lequel, pour Yves Jeanneret, "exprime bien cette superposition du poétique, du théorique et du machinique, [impliquant] que la procédure technique de fragmentation des fichiers rencontre la démarche intellectuelle de l'ouverture du sens ${ }^{13}{ }^{13}$.

Si l'outil multimédia permet bien «une nouvelle configuration de nos pratiques de lecture ${ }^{14}$ », s'il autorise «une délinéarisation du discours narratif auctorial» et «sa relinéarisation par un lecteur singulier $»^{15}$, alors l'hypertexte, avec ou sans -e, est bien le fils spirituel du Texte, avec ou sans majuscule. Lorsque Barthes défend, dans «Les Sorties du texte», que «le désir n'est pas dans le texte par les mots qui le «représentent», qui le racontent, mais par des mots suffisamment découpés, suffisamment brillants, triomphants, pour se faire aimer, à la façon de fétiches $\left.{ }^{16}{ }^{\prime}\right)$, on répondra par le double-clic : ce mot qui sort du fond gris de la page, qui permet de lire les yeux levés du livre, l'opération préalable d'encodage du texte multimédia en a fait une balise active qui envoie

Io. R. Barthes, «Texte (théorie du)», [1973], OC, IV, p. 448.

I I. R. Barthes, «L'aventure sémiologique», [1974], OC, IV, p. 524.

I 2. G. Landow, Hypertext: the convergence of contemporary critical theory and technology, BaltimoreLondres, The Johns Hopkins University Press, I 992.

I 3. Y. Jeanneret, «Les résurrections médiatiques de l'auteur. Médias, médiations, figures», consultable en ligne à l'adresse suivante : http://www.ec-aiss.it/contributi/jeanneret_02_08_05.html.

I 4. B. Gervais, «Naviguer entre le texte et l'écran. Penser la lecture à l'ère de l'hypertextualité», Les Défis..., op. cit., p. 60.

I 5 . J. Clément, «Hypertexte et fiction : une affaire de lien», Les Défis..., op. cit., p. 7I.

I6. R. Barthes, «Les Sorties du texte», [1973], OC, IV, p. 376. 
ou renvoie vers un ailleurs textuel. Dès lors, je citerai cet extrait de l'«Analyse textuelle d'un récit d'Edgar Poe» :

[...] le mode de présence du sens (sauf peut-être pour les séquences actionnelles) n'est pas le développement, mais l'éclat : appel de contact, de communication, positions de contrat, d'échange, éclats des références, des lueurs de savoir, coups plus sourds, plus pénétrants, venus de «l'autre scène», celle du symbolique $[\ldots]^{17}$.

À cette élaboration théorique répond la défense de la technologie hypertextuelle par Jean Clément, pour qui il s'agit d'

[...] une technologie à la fois intellectuelle et énonciative qui rompt avec la linéarité du discours, introduit des ruptures, produit du désordre et du jeu dans les activités d'écriture et de lecture. [Cette technologie] favorise une écriture fragmentaire, elliptique, déliée des règles de la rhétorique traditionnelle» (art. cit., p. 72).

N'est-ce pas là ce que Barthes lui-même, dans l'exposition de son cours, souhaitait mettre en œuvre? Las de ce qu'il nomme le maniement hypocrite des fiches, il élabore en effet une présentation déliée de la rhétorique traditionnelle du plan. Le Vivre ensemble, à partir du fantasme d'idiorrythmie qui fonde sa démarche ${ }^{18}$, se présente ainsi comme l'exposition aléatoire de trente et une figures ordonnées selon l'arbitraire de l'ordre alphabétique : le professeur, au fil des séances, ne travaille pas au lien entre les figures «Animaux» (p. 59-64), «Fleurs» (p. I 25-I 27) ou «Rectangle» (p. I 5 8-I6I), mais pose que toutes, plus ou moins lointainement, peuvent faire écho à l'interrogation portée par le titre de l'enseignement. C'est dans ce principe d'une présentation libre du propos que s'installe le site, puisqu'il propose au lecteur/visiteur de se promener à son aise dans les massifs découpés par le professeur. Libre à chacun, grâce à l'accès offert par chacune des figures, de se laisser porter par la voix du Maitre ou de suivre les parcours que proposent les notes manuscrites.

À cette première liberté de navigation, le site ajoutait une seconde : celle d'un index qui permettait d'accéder directement à certains développements du cours. L'utilisateur pouvait ainsi traquer les entrées attendues dans le cas de Barthes : «signifiant», «texte», «structure»... chacun de ces termes fonctionnait comme autant de voies d'accès au contenu du cours. Une fenêtre s'ouvrait alors à l'écran, proposant un accès direct à tel ou tel passage. Seules

I7. R. Barthes, «Analyse textuelle d'un récit d'Edgar Poe», [1973], OC, IV, p. 440.

I 8. Barthes s'en justifie lors de la première séance : le fantasme du cours, ce «retour de désirs, d'images, qui rôdent, se cherchent en vous», «à l'occasion d'une lecture gratuite (Lacarrière, L'Été grec) a trouvé le mot qui l'a fait travailler» (Comment vivre ensemble, op. cit., p. 3637). Ce mot, idiorrythmie, désigne, comme le rappelle $\mathrm{Cl}$. Coste en préface du cours, «toutes les entreprises qui concilient ou tentent de concilier la vie collective et la vie individuelle, l'indépendance du sujet et la sociabilité du groupe» (p. 25 de l'édition citée). 
les transcriptions des notes et de l'enregistrement sonore étaient indexées, mais ce mode d'accès au cours figurait déjà une liberté de lecture accrue, dans le prolongement de la liberté dont le professeur avait lui-même témoigné. Une telle navigation hypertextuelle semblait réaliser alors, plus de vingt après, le souhait barthésien d'un «tracé excentrique de possibilité», d'un «butinage» parfois ludique, toujours savoureux (CVE, p. г 80).

\section{La poétique effective du cours : effets de dissension}

Mais le cours respecte-t-il ce jeu? Peut-on lire le déroulement des quatorze séances comme soustraction inouïe à la linéarité du développement, et voir dans le texte du cours, conformément au programme annoncé par Barthes à la première séance, un éclatement du discours en une galaxie de menues significations? Il convient de bien tenir compte des dispositifs pragmatiques redoutables des textes de Barthes, dispositifs qui voient souvent les textes déjouer de l'intérieur d'une écriture la théorie qu'ils exposent.

Certes, la première séance du Vivre ensemble s'ouvre sur un souvenir de l'opposition nietzschéenne entre méthode et paideia, c'est-à-dire entre la volonté droite du penseur qui fétichise le but à atteindre, et la liberté d'une réflexion qui activerait le psychisme anti-phallique du voyage... On pourrait alors prêter crédit au cours, et le situer, sur son invite, du côté de la nonméthode, de la paideia : en témoignerait l'arbitraire de l'ordre alphabétique, «qui est, comme chacun le sait, tout à la fois un ordre et un désordre, un ordre privé de sens, le degré zéro de l'ordre» - je cite Barthes dans «Les Sorties du texte $^{19}$ ». Ce degré zéro de l'ordre, où ne pourrait se lire aucun intelligible, répondrait à la volonté de ne souscrire en rien à la «méthode». Pour autant, lors de la dernière séance, à l'occasion d'un retour sur les modalités d'exposition du cours, Barthes reconnaît : «Évidemment, ici, on ne s'est pas placé du côté de la méthode, mais du côté de la paideia, ou pour le dire plus prudemment (et provisoirement) du côté de la non-méthode» (CVE, p. I 80). Retenons la prudence et le provisoire qui s'entrelacent, dans un double motif de la réticence et du non encore advenu qu'il semble falloir garder en mémoire. Car si le cours affiche sa prédilection pour «un protocole d'exposition» inouï qui le préserve de tout ordre dissertatoire, il faut bien lire ce qu'il en dit : Barthes parle d'«un procédé créatif que connaissait bien la peinture chinoise : le hasard contrôlé, le léger contrôle du hasard, dans l'opération de classement : le rangement alphabétique» (ibid., p. I 8 I). Ce «léger contrôle du hasard» doit nous

I9. R. Barthes, «Les Sorties du texte», [1973], OC, IV, p. 366. 
inciter à la prudence et peut fonctionner comme une invite à relire le parcours du Vivre ensemble à la lumière du jeu du classement : le hasard, dès lors que contrôlé, n'aurait-il pas produit un ordre, dissimulé à la sagacité du lecteur, mais pertinent pour l'appréhension du cours? Regardons alors les figures liminaires : elles dessinent un parcours, discret, qui court de l'acédie à la xeniteia, c'est-à-dire, pour en proposer la traduction que donne Barthes, du désinvestissement du moine (l'acédie) à la volonté de «dépaysement», au désir d'«exil volontaire» (la xeniteia) (ibid., p. I7I). Ainsi, comme deux portants d'un arc, l'acédie et la xeniteia permettraient de lire d'abord le rejet du lieu où l'on se trouve pris, et son symétrique inversé, le désir d'un autre lieu. De la première à la dernière séance du cours, on peut donc lire un cheminement, qui serait moins narratif et dissertatif que symbolique ${ }^{20}$. Dès lors, et ce malgré qu'il en ait, le cours ne souscrit pas à la lecture éclatée qu'il propose; plus encore, il utilise la structure narrative de l'incipit et de l'explicit pour y celer un de ses secrets d'écriture.

La structure narrative sous-jacente au cours déconstruit son dispositif d'exposition, et retourne la fatalité de la linéarité de la lecture en outil heuristique. Lecture - hypothèse de lecture, je le concède bien volontiers - que néanmoins le moteur de navigation ne permet pas de laisser apparaittre : faire éclater le texte du cours selon les aléas d'une lecture hypertextuelle, se déplacer à sauts et à gambades dans les trente et une figures en les considérant comme autant de satellites autour de la question du «Vivre Ensemble» parait alors faire violence à un cours qui dissimule son cheminement. Et si donc l'outil hypertextuel semblait le moteur idoine pour un texte éclaté, sans structure, sans disposition, l'obstacle réside dans le programme défaillant d'écriture du cours, qui ne réalise qu'imparfaitement cette galaxie de traits signifiants qu'il promettait et, immanquablement, réintroduit du sens dans une exposition qu'il avait condamnée de prime abord. Dès lors, on pourrait rendre compte de cet échec de la lecture hypertextuelle, échec inscrit dans le texte barthésien lui-même, par cette citation de l'article «Sur la lecture»: «le lecteur est pris dans un renversement dialectique : finalement, il ne décode pas, il sur-code; il ne déchiffre pas, il produit, il entasse des langages ${ }^{21} \gg$. Ainsi, la lecture hypertextuelle ne se fond pas dans le programme du texte barthésien, mais lui surimpose son mode d'appréhension du donné textuel; elle ne réalise pas l'exemplaire lecture délinéarisée à laquelle le cours prétend s'offrir,

20. É. Marty procède à une lecture identique dans le cas des Fragments d'un discours amoureux: considérant les figures liminaires, «S'abîmer» et «Vouloir-saisir», il conclut : «l'alphabet cesse d'être utilisé dans un ordre aléatoire et arbitraire pour dessiner un ordre savant». É. Marty, Roland Barthes. Le Métier d'écrire, Seuil, «Fiction et Cie», 2006, p. 266 et suiv.

21. R. Barthes, «Sur la lecture», [1974], OC, IV, p. 935. 
mais construit son propre parcours. Une telle objection pourrait alors suffire à invalider le bien-fondé d'une publication en ligne des archives du cours. Mais ce serait ne reconnaître au projet éditorial que le vœu pieu de répondre à la liberté du professeur par la libération d'une lecture jusque-là contraire par l'édition papier. Or, il semble bien plutôt que ce soit moins dans la liberté que dans la conscience critique du visiteur que reposait l'intérêt du site.

\section{Une édition en mouvement}

Il ne s'agit pas, en effet, d'envisager cette publication en ligne dans l'ombre portée de l'édition papier: le roland-barthes.com ne se proposait pas comme une extension, une application au goût des dernières technologies des principes qui fondaient la parution papier. Dans le cadre d'une exploitation éditoriale d'abord limitée au support papier comme medium exclusif de diffusion du cours, le recours au Web, et aux possibilités qu'il offre, semble témoigner d'une prise en considération du statut singulier des documents par lesquels est aujourd'hui disponible l'activité enseignante de Barthes.

Car qu'est-ce que ce cours que l'on publie? Est-ce au moins un texte, $d u$ texte? «Production spécifique», dit Barthes dans la première année de La Préparation du roman, "ni tout à fait parole, ni tout à fait écriture ${ }^{22} »$, le cours s'inscrit dans cet «interstice» qu'évoque Emmanuel Souchier ${ }^{23}$, interstice qui inviterait, en compensation, à la multiplication des documents et la concurrence des media. La mise en publicité de cet insituable s'autoriserait donc l'abondance des supports de diverses natures, qui emprunteraient à l'oral autant qu'à l'écrit en préparation. Et c'est bien là, semble-t-il, le principal avantage qu'offre l'édition multimédia : plus que la liberté de parcours singuliers construits par chaque utilisateur, c'est la concurrence des documents relatifs au cours qui fonde la pertinence du recours au site. Sans reprendre les craintes de Yannick Maignien, qui cerne, à propos des différentes mises en lignes, le risque d'une «indistinction dans le statut ou l'intérêt éditorial ${ }^{24}$ », cette coprésence des différents états du cours permet d'esquiver le figement du discours enseignant dans la seule forme offerte dans le cadre de l'édition papier. La transcription des notes autographes utilisées par le professeur n'est en effet pas présentée autrement par les éditeurs que comme compromis, issu

22. R. Barthes, La Préparation du roman, édité par N. Léger, sous la direction d'É. Marty, Seuil, 2003, p. 3I.

23. E. Souchier, «L'exercice de style éditorial : avatars et réception d'une œuvre à travers l'histoire, des manuscrits à Internet», Communication \& langages, $\mathrm{n}^{\circ}$ I 35, 2003, p. 45-72. 24. Y. Maignien, «Vérité et fiction sur internet», Les Défis..., op. cit., p. $27 \mathrm{I}$ 
de l'exigence de respecter les principes mêmes qui organisent la pensée de Barthes. Permettre l'accès au discours enseignant par les notes manuscrites, ou par l'enregistrement sonore revient bien à reconnaître qu'il n'est aucune voie à privilégier - aucune voix non plus. L'édition multimédia, tout en mettant à nu l'impossibilité d'une mise en publicité neutre du cours, permettait au lecteur lui-même de mesurer les opérations effectuées par le travail d'édition. Le pluriel de l'offre, par l'hétérogénéité même des archives à disposition, figurait une chance : celle de ne pas figer le cours dans une seule forme, de rappeler que celle sous laquelle ils sont le plus directement accessibles (l'édition papier) relève d'un parti-pris.

Il convient dès lors de ne pas considérer la publication en ligne, malgré les métaphores évidentes que suggère l'usage courant, comme toile d'araignée, organisée autour d'un centre autant improbable qu'irréalisé - celui de ce «texte» qui toujours se refuse au cours. Bien au contraire, on pourrait penser cette édition comme rhizomes, dérivés autour de l'absence de forme fixe ou stabilisée. En ce sens, l'outil multimédia emporte un avantage qu'il ne faut pas sous-estimer : celui de permettre l'accès au cours par différentes voies, celles du document manuscrit, de l'enregistrement sonore, et de leur transcription respective. Ces différents documents, la présentation en ligne ne travaille pas à les hiérarchiser les uns par rapport aux autres. Bien au contraire, elle les propose comme équivalents les uns des autres, simples traces, toujours lacunaires, d'une activité enseignante à jamais évanouie. Par la richesse de leur offre, ces pages Web voyaient disparaitre le risque du figement du cours en un énoncé attesté, et restituaient la mouvance de son énonciation. Et c'est en ce sens qu'on peut concevoir l'édition en ligne du Vivre ensemble : édition mouvante, qui refuse de choisir entre medium écrit ou oral; édition en mouvement, dirigée vers un visiteur à même de mettre en perspective les documents qui lui étaient offerts.

Il s'agissait bien, par la multiplication des documents relatifs au cours, de briser toute illusion d'empathie : la mise en regard de l'archive sonore et des notes manuscrites travaillait à faire du visiteur un lecteur distant, donc critique. Face aux deux publications existantes du cours (l'édition imprimée et le $\mathrm{CD} \mathrm{MP}_{3}$ ), elle constituait la possibilité d'un lieu autre, où chacune des exploitations éditoriales se révélait lacunaire. Dès lors, la chance du site était dans cette réponse apportée au sentiment du lecteur que le cours lui échappe, qu'il est le fruit d'une situation pragmatique, d'une réalité singulière pour toujours abolie.

Ainsi, au terme de ce parcours d'un site qui n'est plus, notre lecture ne peut que faire sienne une des leçons que tirait Barthes lors des derniers mots du Vivre 
ensemble: «Il n'y a pas de présent : c'est un temps impossible» (CVE, p. 3 I). Et c'est bien la moralité à tirer aussi de notre confrontation des documents : le cours, que Barthes n'a jamais voulu publier, cette "part de monument refusé26" telle qu'il la défend dans La Préparation au roman, figure bien ce présent, cet éphémère. L’ultime enseignement du Vivre ensemble, dans les aléas et les mésaventures de son transfuge en communauté internaute, réside peut-être dans cette tentative de trouver la publicité adaptée à un ensemble d'archives hétérogènes, d'énoncés oraux ou écrits tournant autour d'une énonciation perdue - celle du cours. Tentative avortée, s'originant pourtant dans ce fantasme d'une communauté d'utilisateurs qui, face à une expérience intellectuelle singulière à jamais disparue, auraient cherché à la (re)vivre, ensemble ou non.

25. La Préparation au roman, op. cit., p. 3 I. 